\title{
Nondifferential training of pigeons retards acquisition of subsequent discriminations involving other stimuli
}

\author{
ROBERT J. NEWLIN and DAVID R. THOMAS \\ University of Colorado, Boulder, Colorado 80309
}

\begin{abstract}
In Experiment 1, two groups of pigeons ( $\mathrm{n}=8$ ) were given nondifferential (ND) training with a green keylight and a white vertical line on a dark surround nonsystematically alternated. Two groups $(\mathrm{n}=8)$ received single stimulus (SS) training with the green light only. In Experiment 2, two groups of pigeons $(n=8)$ were given ND training with vertical and horizontal lines, while two other groups $(n=8)$ received SS training with only the vertical line. In both experiments, all groups were transferred to a green $\mathrm{S}+$ (VI reinforced) and a red $\mathrm{S}-$ (extinguished) transfer problem. In each experiment, one ND and one SS group was tested in the same context as initial training (houselight off) and one ND and one SS group was tested in a changed context (houselight on). In both experiments and in both contexts, the ND groups performed less well on the transfer problem than did the SS groups. There was no evidence of greater control by the context in ND than in SS groups, which suggests that the observed difference in acquisition of the transfer task is not attributable to a purported difference in control by the context under the two conditions. The overall results favor the position that nondifferential training reduces attention to stimuli involved in the original training procedure and that this reduced attention transfers to stimuli subsequently experienced.
\end{abstract}

True discrimination (TD) training sharpens subsequent generalization gradients on dimensions other than those specifically involved in the discrimination, relative to either single stimulus (SS) or non differential (ND) training (cf. Honig, 1969; Switalski, Lyons, \& Thomas, 1966; Thomas, Freeman, Svinicki, Burr, \& Lyons, 1970). Similarly, ND training flattens gradients relative to SS training (cf. Bresnahan, 1970; Thomas, Miller, \& Hansen, 1972). Thomas (1970) has interpreted this pattern of results as reflecting a process of general attention. Presumably, when exposed to TD or ND training, subjects learn about the relevance or irrelevance of stimuli (in this experimental situation) in general and not only about the specific training values. This general set, characterized as either an attentiveness or inattentiveness to stimulus differences, may then be reflected in sharper or flatter generalization gradients measured along dimensions which may or may not have been involved in original training. It should be noted that this use of the concept of attention does not refer to the extent to which the subject notices the stimuli, but rather to the degree to which he uses them as the basis on which to determine future responding.

This research was supported by Research Grant HD-03486 to David R. Thomas. It was reported by Robert J. Newlin at the April 1978 meetings of the Rocky Mountain Psychological Association in Denver, Colorado. We wish to thank Leha Robles and James Rodgers for a critical reading of the manuscript. Reprints may be obtained from either author at: Department of Psychology, University of Colorado, Boulder, Colorado 80309.
Indeed, if the subject did not notice the stimuli, he could not learn whether or not they were relevant.

The effects of TD, ND, and SS training have also been studied in a transfer-of-training paradigm. Subjects given TD training acquire a subsequent discrimination task more rapidly than SS- or ND-trained subjects (Eck, Noel, \& Thomas, 1969; Frieman \& Goyette, 1973). This positive transfer has also been attributed to the process of general attention (Eck et al., 1969). Solving a discrimination task may be viewed as, first, attending to the relevant stimuli and, second, attaching the correct responses to these stimuli.

The general attention position predicts that analogous to the relative flattening of slope in generalization following ND training, performance in the acquisition of a new discrimination task should be retarded in ND-trained subjects as compared to SS-trained subjects. Although several experiments have included SS and ND groups (cf. Eck et al., 1969; Sherman, Robles, \& Thomas, 1977; Thomas, Miller, \& Svinicki, 1971), none have reported reliable differences in their transfer performance. There are several possible reasons for this. First, if subjects don't notice the difference between stimuli in training, they will fail to learn that the stimuli are irrelevant. This consideration leads to the prediction that the more different the stimuli in training, the larger the ND effect. Tomie, Davitt, and Thomas (1973) tested this hypothesis with generalization gradients and found that interdimensional training, where the training 
stimuli are from different dimensions, does indeed produce flatter gradients after ND training than does extradimensional training, where the training stimuli are on the same dimension but orthogonal to the test stimuli.

Second,the amount of training in the first stage may be important. Eck et al. (1969) yoked TD and ND subjects in such a way that when TD subjects reached a predetermined discrimination ratio both ND and TD subjects were transferred. If irrelevance learning takes as long on the average or longer than does discrimination learning, then many of the ND birds in the Eck et al. study may not have learned about the irrelevance of stimuli. Perhaps, if more extended training had been given, evidence of irrelevance learning would have been obtained.

The need for more sessions introduces a third problem. Welker, Tomie, Davitt, \& Thomas (1974) have shown that after substantial SS training, acquisition of a subsequent discrimination is severely retarded. They interpreted this retardation as a direct effect of control by context (those elements of the environment which are common to training and transfer). In other words, control by the context acquired in original training may "block" the acquisition of control by stimuli presented on the response key in the transfer phase of the experiment. If the SS group learns very slowly, it may be impossible for the ND group to show even slower learning. There are at least two ways to counteract this "floor" effect. First the test task should be as easy as possible to allow for more rapid learning to occur. Second, a context shift at the time of testing may reduce the context effect so that learning will occur more rapidly. Both of these methods were employed in the present experiments.

The ND-SS comparison is of special interest because several explanations which have attempted to account for the sharpening of TD gradients or the more rapid acquisition of a subsequent discrimination by TD subjects are not readily applicable to this case. Turner and Mackintosh (1972) suggested that the important factor might be response-produced feedback. That is, subjects in the ND condition have been reinforced for responding continuously and the feedback from each response is a stimulus which controls further responding. For TD subjects, the discriminative stimuli control responding and feedback from response is not a reliable cue for further responding. This tendency to continue responding would explain the difference between TD and ND subjects in either gradients or transfer tasks; however, this explanation cannot account for the difference between ND and SS subjects, both of whom have been trained to respond continuously. Goyette and Frieman (1973) suggested that the TDND difference may be due to the fact that TD subjects have learned to withhold responding, which can then be applied to the new discrimination yielding higher discrimination ratios. Again, this does not apply to the ND-SS case where neither group has been trained to withhold responding.

Mackintosh (1977) has presented a selective attention interpretation of the effects of TD, SS, and ND training. According to this view, the context acquires differential control in these three procedures. For TD, the discriminative stimuli gain most control and the context gains little. In ND, the irrelevant stimuli gain little control and the context gains major control over responding. For SS training, both the stimulus and context gain moderate amounts of control over responding. In generalization testing, the more control the context exerts, the less is available to stimuli manipulated by the experimenter, thus the flatter the generalization gradient. In transfer, the control by context blocks acquisition of control by the new discriminative stimuli. If this view is correct, then a context change between training and transfer testing should remove more control for ND than for SS subjects, because the context has more control for ND subjects. This should reduce any ND-SS difference which might otherwise be present.

In Experiment 1, we used the interdimensional paradigm which Tomie et al. (1973) had demonstrated was the most effective in producing ND and SS generalization gradient differences. Also, for half of the subjects in each group, the transfer context was different from the training context in an attempt to determine whether control by context mediates the transfer effect or blocks it.

\section{EXPERIMENT 1}

\section{Subjects \\ Method \\ The subjects were 32 experimentally naive adult pigeons ob- tained from a local supplier and maintained at approximately $75 \%$ of their ad-lib weights throughout the experiment in a colony with a 16-h light/8-h dark cycle.}

\section{Apparatus}

Four standard pigeon chambers were used (cf. Hickis, Robles, \& Thomas, 1977). On-key discriminative cues were provided by IEE (Series 0010) in-line display cells mounted $.635 \mathrm{~cm}$ behind the key. Display cells fitted with Kodak Wratten filters No. 99 and No. $72 \mathrm{~B}$ provided chromatic stimuli with nominal peak wavelength transmission of $555 \mathrm{~nm}$ (green) and $606 \mathrm{~nm}$ (red), respectively. The display cell allowed projection of a white line, $.32 \mathrm{~cm}$ wide and $2.5 \mathrm{~cm}$ long, on a black surround in either a vertical or a horizontal position. Rear-projection of these stimuli on the pecking key was accomplished by the illumination of miniature 14-V lamps (No. 1815). Chamber illumination could be provided by a $28-\mathrm{V}$ miniature lamp (No. 1829) centered behind a $2.4-\mathrm{cm}$ high strip of white Plexiglas extending the width of the chamber above the intelligence panel.

\section{Procedure}

Preliminary training. On Day 1, all pigeons were hand-shaped 
to keypeck for $3 \mathrm{sec}$ of access to mixed grain. Following the continuous reinforcement of the first 20 responses, the next 40 reinforcers were earned on a gradually increasing variable-interval (VI) schedule of reinforcement until a VI 1-min schedule was in effect. In the next session, pigeons earned 50 reinforcers on the same gradually increasing VI schedule. During this training, the houselight was not illuminated and the key was illuminated green.

Stage I. During Stages I and II, the subjects received daily training sessions of $30 \mathrm{~min}$ duration. Pigeons were assigned to four groups $(n=8)$. Groups SS and SSC $(C=$ context change $)$ received training with only the green key. Groups ND and NDC were presented with alternating 1-min periods of green key or white vertical line on the key. During this training and the subsequent transfer task, there were three blocks of 10 stimulus presentations each per session. Within each block, each stimulus was presented five times with no more than 3 consecutive minutes of any one stimulus. For all groups, a VI 1-min schedule was in effect at all times and reinforcers not collected were cancelled at the end of each 1 -min period. Training was continued for 20 sessions. The houselight was not illuminated during this stage.

Stage II. During Stage II, all groups were given discrimination training with green as $\mathrm{S}+$ and red as $\mathrm{S}-$. For Groups NDC and SSC, the houselight was illuminated; for Groups ND and SS, it was not. The VI 1 -min schedule was in effect only during $\mathrm{S}+$ periods. Training was continued for 10 sessions.

\section{Stage I}

\section{Results}

The mean number of responses per session (averaged over the last five sessions of Stage I) emitted to the green key by the ND and NDC groups were 916.8 and $1,130.7$, respectively, and to the vertical line were 829.4 and $1,092.8$. The mean responses for comparable periods of the sessions for the SS and SSC groups were 990.0, 1,365.3, 974.5 and $1,362.4$. One subject in the SSC group failed to maintain responding after the context change and was dropped from this and subsequent analyses.

On the first day of ND training, ND and NDC subjects discriminated between the two stimuli with all but 1 of the 16 subjects responding more to the green stimulus used in shaping than to the vertical line $(p<.01$, binomial test, two-tailed). Mean discrimination ratios (i.e., percent of total responses to green) on the first day of ND training were 76 and 59 for the ND and NDC groups, respectively. On the last day of ND training, they were 53 and 51 , with 7 of the 16 subjects responding more to green than to vertical.

\section{Stage II}

As was expected, learning was slow; only the SSC group exceeded a $90 \%$ discrimination ratio. Figure la presents the discrimination ratios for the four groups for the 10 sessions of discrimination training. A 2 by 2 by 10 analysis of variance indicated a reliable effect for sessions $[F(9,243)=4.71, p<.01]$, an effect for context change $[F(1,27)=7.34, p<.02]$, and an effect for ND-SS $[F(1,27)=8.64, \mathrm{p}<.01]$ but no interaction term was significant (all $\mathrm{Fs}<1.0$ ).

Analysis of the rates to $S+$ (Figure $1 b$ ) indicated no main effects of group treatments (Fs $<1.0)$, but a highly reliable effect for increase over the 10 sessions and a highly reliable interaction between increase and context change $[F(9,252)=18.35$ and 8.04 , respectively, both ps $<.01$; i.e., the groups for which context was changed began responding at a lower rate and ended at a higher one.
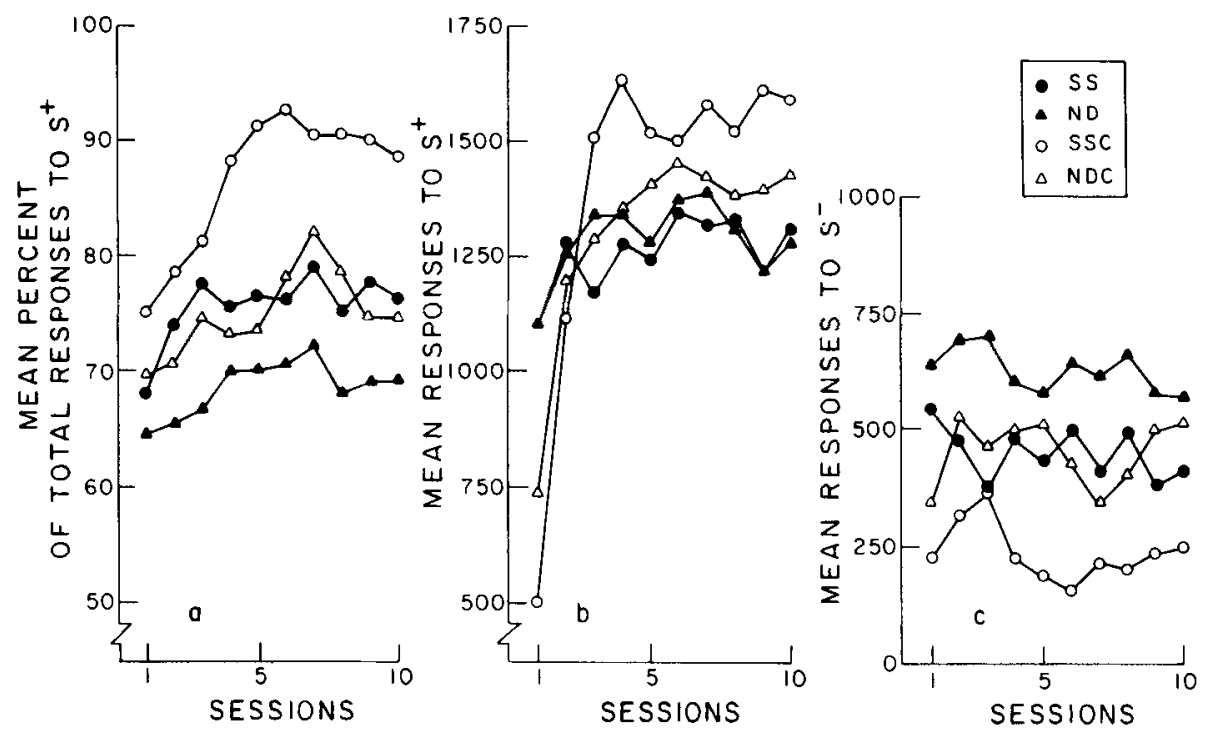

Figure 1. (a) Mean percent of lotal responses to $S+$, (b) mean total responses to $S+$, and (c) mean total responses to S - , in the SS, ND, SSC, and NDC groups in Experiment 1. 
Analysis of $\mathrm{S}-$ rates (Figure $1 \mathrm{c}$ ) revealed a reliable effect for ND-SS $[\mathrm{F}(1,27)=6.08, \mathrm{p}<.05]$ and for context $[F(1,27)=5.98, p<.05]$. The effect for sessions was not reliable, nor was the interaction between sessions and context $(\mathrm{Fs}<1.0)$.

\section{Discussion}

The results of Experiment 1 clearly demonstrate the retardation of the acquisition of a discrimination by ND-trained subjects relative to those given SS training. It is interesting to note that the poorer performance of the ND subjects was a consequence of their responding at a higher rate to $S$ - rather than their responding less to $S+$. This parallels the finding that the TD facilitation effect is usually mediated through more rapid reduction of responding to S - (cf. Eck et al., 1969).

The dramatic initial reaction of responding to both $\mathrm{S}+$ and $\mathrm{S}-$ in the context-change groups indicates that contextual cues did, indeed, exert considerable control over responding. Upon removal of that control, the discriminative stimuli should gain more control, and they did: under both ND and SS conditions, the context-change groups had higher discrimination ratios than the no-context-change groups. However, there was no evidence that the context had more control in the ND than in the SS groups.

In the interdimensional paradigm, there is a stimulus common to both stages of training (in this case, the green key). Although a mechanism has not been suggested, it is possible that poor performance in discriminating red from green could be mediated by what was learned about green in the first stage. Evidence for a more general process of inattention would require that the discriminative stimuli used in Stage II be orthogonal to those used in nondifferential training in Stage I. Although the extradimensional design has not succeeded in revealing an ND retardation effect in previous studies, we have never used the green-red transfer problem which was successful in the case of interdimensional training, nor have we used the context change manipulation in an extradimensional transfer experiment. In Experiment 2, we did both of these things.

\section{EXPERIMENT 2}

\section{Method}

Subjects

The subjects were 32 experimentally naive pigeons, maintained as in Experiment 1.

\section{Apparatus}

The apparatus was the same as that used in Experiment 1 .

\section{Procedure}

Preliminary training. Pigeons were divided into ND, NDC, SS, and SSC groups $(\mathrm{n}=8)$, as in Experiment 1 . On Days 1 and 2, all pigeons were hand-shaped to peck the green key and the VI schedule gradually increased as in Experiment 1, with the exception that for Groups ND and SS the houselight was not illuminated and for Groups NDC and SSC it was. A third day was added to this stage, in which all birds received 50 reinforcements on the increasing VI schedule for pecking the vertical line, with the houselight not illuminated. This preliminary training was necessary in order to maintain pecking in Stage II, when both keylight and houselight were changed simultaneously.

Stage I. This stage was identical to Stage I of the previous experiment except that for the SS and SSC groups the vertical line was on the key and for the ND and NDC groups the vertical line and a horizontal line alternated. This stage lasted for 20 sessions.

Stage II. This stage was identical to Stage II of Experiment 1 and lasted for 10 sessions

\section{Stage I}

\section{Results}

The mean number of responses per session (averaged over the least five sessions of Stage I) emitted to the vertical line by the ND and NDC groups were 862.3 and 779.5 , respectively, and to the horizontal line were 856.8 and 808.1 . The mean responses for comparable periods of the sessions for Groups SS and SSC were 770.5, 1,056.9, 764.7, and 1,052.9.

On the first day of ND training, ND and NDC subjects discriminated between the two stimuli, with all but 2 of the 16 subjects responding less to the horizontal line than to the vertical line stimulus used in the previous training session $(p<.01$, binomial test, two-tailed). Mean discrimination ratios on the first day of ND training were 53 and 56 for the ND and NDC groups, respectively. On the last day of ND training, they were 51 and 48, with 7 of the 16 subjects responding more to the vertical line than to the horizontal line.

\section{Stage II}

Again, learning was relatively slow (Figure 2a). Mean discrimination ratio showed an effect for NDSS $[F(1,28)=6.51, p<.02]$, but no effect for the context change ( $F<1.0)$; nor was there an interaction between context change and ND-SS $(F<1.0)$. There was a significant trend over the 10 sessions $[F(9,252)=5.95, p<.01]$ and a significant effect for ND-SS by Sessions $[F(9,252)=2.30, p<.02]$, but no effect for Context by Sessions $[F(9,252)=$ $1.01, \mathrm{p}>.10]$. Finally, there was a significant interaction between sessions, ND-SS, and context $[\mathrm{F}(9,252)$ $=2.00, \mathrm{p}<.04]$.

Analysis of the rates to $S+$ (Figure $2 b$ ) indicated no effect of context $(F<1.0)$ or ND-SS $[F(1,28)=$ $1.33, \mathrm{p}>.10]$ or any interaction $(\mathrm{F}<1.0)$, but a reliable effect for sessions $[F(9,252)=11.72, p<$ $.01]$ and no interactions between sessions and context or ND-SS $[\mathrm{F}(1,252)=1.43, \mathrm{p}>.10$ and $\mathrm{F}<1.0$, respectively]. The three-way interaction was not significant $[\mathrm{F}(1,252)=1.23, \mathrm{p}>.10]$. 

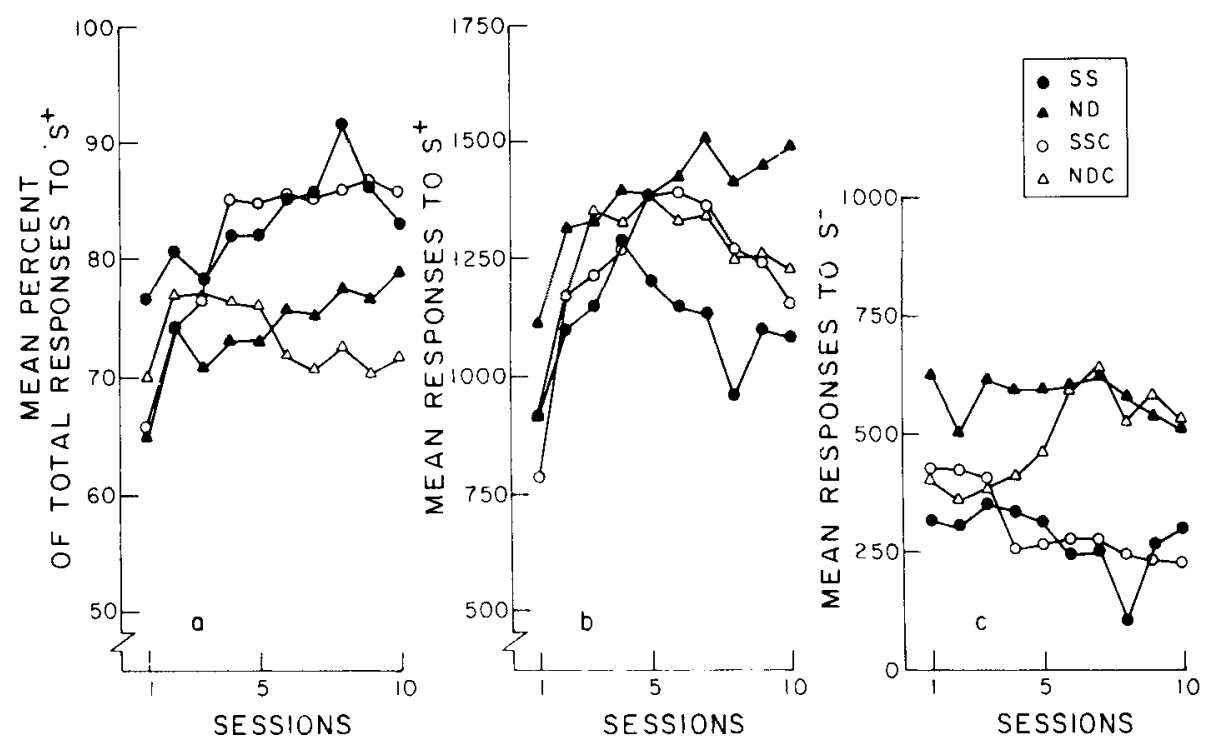

Figure 2. (a) Mean percent of total responses to $\mathbf{S}+$, (b) mean total responses to $\mathbf{S}+$, and (c) mean total responses to S - , in the SS, ND, SSC, and NDC groups in Experiment 2.

Analysis of the $\mathrm{S}$ - rates (Figure $2 \mathrm{c}$ ) indicated no effect for context change $(F<1.0)$, but a reliable effect for ND-SS $[F(1,28)=5.55, \mathrm{p}<.03]$, and no interaction $(F<1.0)$. There was no effect for sessions or Sessions by Context (Fs $<1.0)$, but a significant interaction between ND-SS and sessions $[\mathrm{F}(9,252)=$ $2.76, \mathrm{p}<.01]$. The three-way interaction was not significant $[F(9,252)=1.34, p>.10]$.

\section{Discussion}

In both experiments, an effect for ND-SS was found as predicted by general attention theory (Thomas, 1970). In Experiment 1, it was possible to attribute this difference to specific control by the green-key stimulus common to both Stages I and II. When this source of control was eliminated in Experiment 2, the effect did not diminish. This suggests that specific learning involving the green stimulus was not responsible for the ND retardation effect in Experiment 1. Further, a context manipulation which had a marked effect on discrimination performance in Experiment 1 failed to reduce the size of the ND-SS effect in that experiment and failed to have an effect at all in Experiment 2. In Experiment 2, the two sessions of pretraining in the context in which transfer was later tested not only served to maintain responding after transfer, but apparently eliminated the effect of the context change. In neither experiment was there any evidence that control by context was greater for the ND groups than for SS groups. Although Mackintosh (1977) suggests that a finding of no differential effect of context change would not contradict his theory since other unmanipulated elements of the context might have differential control, it would seem un- reasonable to postulate greater control by unspecified contextual cues following ND training when, in Experiment 1, changing a specifiable visual context with demonstrable control failed to show differential control. Indeed, these studies suggest that the context plays less of a role in transfer than Mackintosh's view would dictate.

Because several aspects of procedure were changed between the present experiments and those which had previously failed to reveal an ND-SS difference, it is not possible to pinpoint which changes or combination of changes account for our present success. However, one point seems particularly relevant in this regard. In the present two experiments, the stimuli used in ND training were sufficiently different for the subjects to initially show a significant preference for that stimulus which had been present in initial shaping. Nondifferential reinforcement soon eliminated this preference; however, the fact that it was initially present demonstrates that the subjects did notice that the stimuli were different such that they could later learn that the stimulus difference was without consequence. In the Sherman et al. (1977) experiment, for example, the training stimuli were lines differing by only $30^{\circ}$ and there was no indication that at any point the subjects noticed that there were two training stimuli rather than one. If this was, indeed, the case, it is not surprising that these subjects had not learned about stimulus irrelevance.

In conclusion, in both of these experiments the ND group showed retarded acquisition of a discrimination relative to an SS group, and in both cases that retardation was mediated by more responding to $S-$. The result was the same in an interdimensional 
transfer task (Experiment 1) and an extradimensional task (Experiment 2). It is therefore not attributable to anything learned about the specific stimulus values used in Stage I. Furthermore, when a context change between stages of training was effective in producing more rapid acquisition of the discrimination (Experiment 1), the magnitude of the ND-SS difference was not affected, arguing against Mackintosh's (1977) suggestion that ND training leads to more control by the context than does SS training, with less control by discriminative stimuli as a consequence of blocking by contextual control. Since other explanations of the TD-ND difference (e.g., response produced feedback and learning to withhold responding) are not applicable to the ND-SS comparison, the results of the three-part TD-SS-ND difference are more readily interpretable as direct reflections of a general process of heightened or reduced attention to stimulus differences as a consequence of discrimination or nondifferential experiences, respectively.

\section{REFERENCES}

Bresnahan, E. L. Effects of extradimensional pseudodiscrimination training upon stimulus control. Journal of Experimental Psychology, 1970, 85, 155-156.

Eck, K. O., Noel, R. C., \& Thomas, D. R. Discrimination learning as a function of prior discrimination and nondifferential training. Joumal of Experimental Psychology, 1969, 82, 156-162.

Frieman, J., \& Goyette, C. H. Transfer of training across stimulus modality and response class. Joumal of Experimental Psychology, 1973, 97, 235-241.

Goyette, C. H., \& Frieman, J. Transfer of training following discrimination learning with two reinforced responses. Learning and Motivation, 1973, 4, 432-444.
Hickis, C. F., Robles, L., \& Thomas, D. R. Contextual stimuli and memory retrieval in pigeons. Animal Learning \& Behavior, 1977. 5, 161.168.

Honig, W. K. Attentional factors governing the slope of the generalization gradient. In R. M. Gilbert \& N. S. Sutherland (Eds.), Animal Discrimination Learning. London: Academic Press, 1969.

Mackintosh, N. J. Stimulus control: Attentional factors. In W. K. Honig \& J. E. R. Staddon (Eds.), Handbook of operant behavior. Englewood Cliffs, N.J: Prentice-Hall, 1977.

Sherman, J. E., Robles, L., \& Thomas, D. R. Asymmetry of training effects in nonspecific transfer of training. Bulletin of the Psychonomic Society, 1977, 10, 165-168.

Switalski, R. W., Lyons, J., \& Thomas, D. R. The effects of interdimensional training on stimulus generalization. Journal of Experimental Psychology, 1966, 72, 661-666.

Tномаs, D. R. Stimulus selection, Attention, and related matters. In J. H. Reynierse (Ed.), Current issues in animal learning. Lincoln: University of Nebraska Press, 1970.

Thomas, D. R., Freeman, F., Svinicki, J. G., Burr, D. E. S., \& Lyons, J. The effects of extra-dimensional training on stimulus generalization. Joumal of Experimental Psychology, 1970, 83, Whole No. 1, Pt. 2, 1-21.

Thomas, D. R., Miller, J. T., \& Hansen, G. Role of stimulus comparison in equivalence training. Journal of Experimental Psychology, 1972, 96, 297-300.

Thomas, D. R., Miller, J. T., \& Svinicki, J. G. Non-specific transfer effects of discrimination training in the rat. Journal of Comparative and Physiological Psychology, 1971, 74, 96-101.

Tomie, A., Davitt, G. A., \& Thomas, D. R. The role of stimulus similarity in equivalence training. Journal of Experimental Psychology, 1973, 101, 146-150.

Turner, C., \& Mackintosh, N. J. Stimulus selection and irrelevant stimuli in discrimination learning by pigeons. Journal of Comparative and Physiological Psychology, 1972, 78, 1-9.

Welker, R. L., Tomie, A., Davitt, G. A., \& Thomas, D. R. Contextual stimulus control over operant responding in pigeons. Journal of Comparative and Physiological Psychology, 1974, 86, 549-562.

(Received for publication January 10, 1978; revision accepted April 24, 1978.) 